\title{
La manipulación en la traducción de noticias del español al inglés. Estudio de caso desde el análisis crítico del discurso'
}

\section{Resumen}

En este artículo se analiza la traducción de una noticia procedente del periódico The New York Times que se ha publicado tanto en inglés como en español, con el fin de delimitar la presencia de manipulación en la traducción para la prensa en contextos políticos. Para ello, se utiliza como acercamiento metodológico el análisis crítico del discurso, en particular, el triángulo ideológico de Van Dijk y las estrategias de manipulación en traducción. Los resultados muestran que la inclusión del análisis crítico del discurso en el análisis traductológico de noticias políticas resulta fundamental para hallar marcas de manipulación.

Palabras clave: traducción, análisis crítico del discurso, manipulación, medios de comunicación, política.

\section{Manipulation in News Translated From Spanish to English. A Case Study From a Critical Discourse Analysis Approach}

\begin{abstract}
In this paper we analyse the translation of a piece of news from The New York Times that has been published in both English and Spanish, in order to identify manipulation in journalistic translation in political contexts. To this end, we use the methodological framework of Critical Discourse Analysis, in particular Van Dijk's ideological triangle, and the strategies of manipulation in translation. Results confirm that the inclusion of Critical Discourse Analysis into the traductological analysis of political news is essential to investigate manipulation.
\end{abstract}

Keywords: translation, Critical Discourse Analysis, manipulation, mass media, politics.

La manipulation dans la traduction de nouvelles de l'espagnol vers l'anglais. Une étude de cas d'après l'Analyse critique du discours

\section{Résumé}

Dans cet article, nous analysons la traduction d'une nouvelle de The New York Times qui a été publiée en anglais et en espagnol, afin d'étudier la présence de la manipulation dans les traductions pour la

1 La presente contribución forma parte de la tesis doctoral que lleva a cabo actualmente la autora en el marco de su contrato para la Formación del Profesorado Universitario (FPU) en la Universidad de Córdoba. Dicha investigación está respaldada económicamente por el Ministerio de Universidades de España (proyecto FPU 19/03266, “Transferencia cultural y discurso político”, año 2019). 
presse dans des contextes politiques. À cette fin, nous utilisons le cadre méthodologique de l'Analyse critique du discours, en particulier le triangle idéologique de Van Dijk ainsi que les stratégies de manipulation dans la traduction. Les résultats confirment que l'inclusion de l'Analyse critique du discours dans l'analyse traductologique de nouvelles politiques est essentielle pour étudier la manipulation.

Mots-clés : traduction, Analyse critique du discours, manipulation, médias, politique. 


\section{Introducción}

Diferentes investigadores han analizado el texto periodístico desde el punto de vista de la traducción (véanse, entre otros, los estudios de Davier y Van Doorslaer, 2018; Gallardo, 2005; Hernández Guerrero, 2008, 2019; Rovena y Marchan, 2017; Schäffner y Bassnett, 2010; Toury, 1985; Tymoczko y Gentzler, 2002; Van Doorslaer, 2010; Wang, 2019). Algunos, en particular, se han ocupado de estudiar la traducción de noticias publicadas por el mismo u otro periódico o agencia de información, en donde es posible hallar enunciados dispares que no siempre cumplen con los criterios más rigurosos relativos a la correspondencia y equivalencia semántica (y formal).

Por ejemplo, en Hernández Guerrero (2019) y Schäffner y Bassnett (2010), se incluye la posibilidad de que los cambios sin correspondencia entre el texto origen (Tо) y el texto meta (TM) de una noticia se deban a factores económicos derivados de los intereses comerciales del propio periódico que el traductor se ha visto obligado a respetar. Así, existen casos en los que la información que se traduce cambia ligeramente de posición ideológica al publicarse en una fuente periodística con distinta orientación política que en la que aparece el to (algo que también vemos en Hernández Guerrero, 2006, o en Ghignoli y Montabes, 2014). Lo que subyace, por ende, es la posibilidad de conectar el ejercicio de la traducción con el de la manipulación ideológica.

Como advierten algunas publicaciones relativas a la manipulación y la traducción (Gallardo, 2005; Toury, 1985; Van Doorslaer, 2010), las noticias políticas que se traducen, dada su naturaleza sensible a la ideología (Van Dijk, 2009), son susceptibles de ser manipuladas durante el trasvase traductológico, tanto por cuestiones estilísticas como por otros factores de índole social y diplomática (Tymoczko y Gentzler, 2002).

Para ilustrar hasta qué punto puede ser determinante la manipulación ideológica en traducción, la pretensión de este artículo es analizar la traducción de una noticia de corte político publicada en español el día 21 de enero de 2017 en el periódico The New York Times: "Trump, un Chevy y la incertidumbre de los cubanos" (Padura, 2017a) y que fue traducida posteriormente, el 31 de enero de ese mismo año, en la versión en inglés de este mismo periódico (Padura, 2017b). Para ello, hacemos uso del triángulo ideológico de Van Dijk (1998) a partir de las premisas teóricas del análisis crítico del discurso (ACD), con el fin de describir el to de la noticia en español y comprobar la pertinencia de las decisiones traductológicas llevadas a cabo para transmitir el mismo sentido de la noticia en la lengua meta (LM) (inglés).

Además, desde el punto de vista traductológico, se incluye el estudio de las estrategias de traducción aplicadas en contextos políticos descritas en Abdi (2019), que a su vez recupera las microestrategias traductológicas de Schjoldager (2008). Estas estrategias se aplican en un estudio enmarcado en el contexto institucional y político.

Este artículo se enmarca dentro de un proyecto de investigación para el que se ha compilado un corpus de trabajo conformado por 56 noticias de corte político internacional, en la combinación de lenguas inglés/español y viceversa, halladas en la prensa. En concreto, hemos acudido a periódicos de todas las ideologías de países de América Latina y Estados Unidos (p. ej., Washington Hispanic, El Nuevo Heraldo, El Chicago Hispano, La Estrella, The Wall Street Journal, usA Today, The New York Times, etc.).

En las páginas siguientes, se resume el estado de la cuestión a partir de los planteamientos teóricos que versan sobre la traducción y la manipulación en los medios de comunicación. Posteriormente, se indica la metodología de trabajo llevada a cabo para analizar las noticias de nuestro proyecto de investigación (enfocada al análisis crítico del discurso) y se aplica, en concreto, en la noticia sobre la que versa este artículo. Finalmente, se enuncian las conclusiones provisionales derivadas de este estudio. 


\section{Traducción, manipulación y medios de comunicación}

Actualmente, el ejercicio de la traducción impera en un buen número de agencias de información, puesto que, dado el mundo globalizado en el que vivimos, muchas de las noticias que leemos, aun sin darnos cuenta, proceden de una noticia anterior que se ha publicado en otro medio periodístico en otra lengua.

Para trasladar el contenido de una noticia a otro idioma, es preciso recurrir a la traducción, si bien en ocasiones resulta complicado localizar una noticia traducida en la que el nombre del traductor aparezca de forma evidente. En muchas ocasiones, se prefiere generalizar y escribir "agencias", para camuflar la realidad de que no se trata de un trabajo de campo del periodista, sino del trabajo de un traductor que colabora para la agencia de noticias en cuestión.

Esto no solo tiene consecuencias para la interpretación del texto en sí, sino también para el análisis de la manipulación de las noticias traducidas en prensa, pues resulta difícil poder garantizar cuándo nos hallamos ante una noticia procedente de una traducción y cuándo no. Como expresan Davier y Van Doorslaer (2018):

In the more frequent cases where the source text is hardly traceable, an analysis of shifts is still possible, but it means that scholars will have to undertake time-consuming detective work and stay at the level of hypotheses in the best-case scenario [...]. In those cases, we believe that the comparability criteria used to collect and build corpora need to be explained in very rigorous and transparent ways [...]. (p. 253). ${ }^{2}$

2 "En la mayoría de los casos en los que es difícil localizar el texto origen, es posible llevar a cabo un análisis de las modificaciones realizadas, pero ello implica que los expertos deberán acometer un trabajo detectivesco al que habrá que dedicarle mucho tiempo y que deberán mantenerse en el nivel de la hipótesis en el mejor de los escenarios [...]. En dichos casos, consideramos que los criterios de equivalencia utilizados
En un contexto de traducción periodística, en el que se parte del supuesto de que muchas noticias que leemos son fruto de distintos procesos de traducción encadenados (Hernández Guerrero, 2019), resulta fundamental identificar y analizar las posibles marcas de manipulación que pueden haberse interpuesto entre el to y el TM, en cuyo proceso de traslación se sobreentiende que se han debido tomar un conjunto de decisiones que dependen de factores como: 1) la orientación política o ideológica del medio donde se publica; 2) la modificación de la información (adición, supresión, etc.) para "domesticar" el TM y evitar elementos extraños en la LM (procedimiento de "domesticación", Hurtado, 2001); y 3) motivos de índole editorial, comercial o incluso de relaciones diplomáticas entre aquellos que tienen la potestad para decidir qué se traduce, qué se publica, cómo se traduce y cómo se publica en el periódico en cuestión.

Por otra parte, lo que se busca al traducir una determinada noticia, especialmente en la era digital, es aumentar el número de lectores. En palabras de Hernández Guerrero (2008):

Las empresas periodísticas en soporte electrónico que buscan su crecimiento y el aumento de su área de influencia crean ediciones digitales en otras lenguas que les permiten rentabilizar la información que producen mediante su traducción, o bien se sirven de modelos mixtos donde la traducción se combina con alianzas mediáticas entre grupos de comunicación afines: las fórmulas de asociación y de producción en el entorno digital son numerosas (p. 88).

Aunque la traducción se conciba en estos tiempos recientes como una estrategia más en el arsenal de posibilidades del periodista (Hernández Guerrero, 2008, p. 88), debemos velar por que se respeten las competencias que definen nuestra especialidad, abogando siempre por la figura del traductor como medio ideal para garantizar

para localizar y crear corpora deberían explicarse en términos muy rigurosos y transparentes [...]" (Trad. propia). 
la calidad que debería esperarse en el proceso traductor de una noticia. Sin embargo, en la práctica, la actividad del periodistatraductor es muy frecuente en aquellos países donde se habla más de una lengua, como ocurre, por ejemplo, entre América Latina y América del Norte, donde hay una vasta cantidad de hispanohablantes y angloparlantes. De ahí que muchos periódicos ofrezcan versiones en línea en varios idiomas (especialmente, en inglés y español).

No obstante, cuando se añade información que no aparece en la versión original y que se aleja del sentido del TO, ya no nos encontramos ante una traducción; en ese caso, más bien nos hallamos ante una edición nueva de la noticia, que se basa en un texto anterior escrito en otro idioma.

Bassnett y Trivedi hacen algunas reflexiones a propósito del ejercicio traductológico, donde se concibe al traductor como un manipulador natural del To:

Para otros, finalmente, traducción es sinónimo de manipulación: [...] La traducción es una actividad altamente manipuladora que involucra todas las etapas del proceso de transferencia a través de fronteras lingüísticas y culturales (Bassnett y Trivedi, 1999, citados en Bastin et al., 2004, p. 71).

De esta cita se colige que la manipulación es una actividad inevitable en el proceso de traducción, dentro de los parámetros gramaticales, estilísticos y editoriales que son necesarios para poder trasladar el mensaje del To al TM. Estos cambios responden casi siempre a cuestiones puramente lingüísticas, pero modifican de cierto modo la forma del To, razón por la que Bassnett y Trivedi (1999) afirman que, en el fondo, el traductor "manipula" el texto de partida.

No obstante, tal "manipulación", siempre entendida enlos términos que acabamos decomentar, no convierte al traductor en manipulador (desde el punto de vista ideológico), sino que simplemente justifica la necesidad de alterar ciertos componentes del то como una parte imprescindible del proceso traductor. Por ende, no toda alteración realizada sobre el To implica una manipulación ideológica en el TM en términos de la escuela de la manipulación, ni siquiera cuando trabajamos con textos traducidos para la prensa - de los que en diferentes estudios se ha demostrado que las marcas de manipulación halladas en los mismos están motivadas por cuestiones ideológicas, políticas o editoriales (véanse, entre otros, Davier y Van Doorslaer, 2018; Schäffner y Bassnett, 2010, y Zhang y Lyu, 2019)-.

En torno a la escuela de la manipulación, resulta obligatorio mencionar a André Lefevere (2004), quien afirmó que "Translation is, of course, a rewriting of an original text" (p. 3). ${ }^{3}$ En los años ochenta, Lefevere presentó un nuevo prisma en los estudios de traducción: el de la manipulación, concepto que desarrolló plenamente en su escuela, al concebir que la práctica de la traducción es el resultado de una manipulación de la ideología y de la crítica literaria sobre el To. Para Lefevere, toda reescritura implica un cierto grado de ideología y de crítica, por lo que se entiende que, para que la literatura funcione dentro de la lengua y la cultura de la sociedad meta, es posible tener que readaptar, modificar y, por lo tanto, "manipular" el To.

A pesar de que la trayectoria de Lefevere comenzó con los estudios de literatura, los tres conceptos fundamentales del desarrollo de su teoría (mecenazgo, ideología y crítica literaria) se han ido abordando desde varias perspectivas de estudio, y en España, en concreto, son muchos los investigadores que han decidido hacerlo desde el punto de vista de la traductología (especialmente, en el contexto de traducción literaria y periodística).

En este sentido, Schäffner y Bassnett (2010) refieren, a propósito de la relación existente entre los medios de comunicación y la traducción (periodística):

3 "La traducción es, indudablemente, una reelaboración de un texto original" (Trad. propia). 
As we saw above, recontextualisation always involves transformation, determined by goals, values and interests. The same holds true for recontextualisation which involves translation. All processes, starting from a decision to report on affairs and events in another country (not only political affairs, but any topic) up to the production of a final text are determined by institutional policies and ideologies. Mass media enable communication across languages and cultures, but in doing so, they can privilege specific information at the expense of other information, and they can also hinder and prohibit information from being circulated (p. 8). ${ }^{4}$

Se da por hecho, por ende, que en la relación entre medios de comunicación, prensa y traducción, pueden llegar a favorecerse determinados procedimientos a través de los cuales se consigue resaltar algunos aspectos de una noticia por encima de otros, que si bien "manipulan" el texto, no tienen por qué deberse siempre a criterios ideológicos.

Para evidenciar la manipulación en las estrategias de traducción es posible utilizar el marco teórico del ACD de Teun A. van Dijk, un enfoque que, aplicado al campo de la traductología, puede ayudarnos a interpretar el sentido vertido sobre el TM y compararlo con el del TO, con el fin de verificar la correspondencia formal y

4 "Como se ha indicado anteriormente, toda recontextualización implica siempre una transformación, determinada por unos objetivos, valores e intereses. Lo mismo se aplica en el caso de las recontextualizaciones que envuelven el proceso de traducción. Todos los procedimientos, empezando por la decisión de informar acerca de acontecimientos y hechos que suceden en otro país (no solo de asuntos políticos, sino de cualquier ámbito) que tienen que ver con la producción del texto final están determinados por políticas institucionales e ideologías. Los medios de comunicación de masas favorecen la comunicación más allá de las lenguas y las culturas, pero al hacerlo, pueden privilegiar cierta información específica en detrimento de otra, y también pueden obstaculizar y prohibir que se propague una determinada información" (Trad. propia). semántica y su posible relación con la manipulación desde el punto de vista del triángulo ideológico. A través del ACD, se busca localizar posibles marcas de manipulación en el transcurso del proceso traductológico, especialmente en textos que requieren de reformulaciones para ser adaptados a la LM y a las políticas editoriales del medio en el que se publican.

Para Fairclough (2006), es importante matizar que, en la traducción de noticias, algunos cambios se justifican teniendo en cuenta el marco del discurso en el que se inserta el to: "When events are reported in news narratives, their form and meaning are transformed according to the genre conventions of news narratives" (Fairclough, 2006, p. 98)..$^{5}$ En ocasiones, el traductor debe presuponer qué información en concreto puede ser sobreentendida por el destinatario meta, de modo que no sea necesario tener que incluirla en el TM. En otras ocasiones, será imprescindible añadir explicitaciones para ayudar al lector meta a entender la información contextual que, por otra parte, se presupone para el destinatario del to.

Así, el triángulo ideológico de Van Dijk, detallado en el siguiente apartado, se configura como un buen instrumento para determinar hasta qué punto la información contenida en el то es suficiente para expresar el sentido del mensaje en la sociedad meta a la que va dirigida la traducción.

La identificación de los conocimientos cognoscitivos propios de la capa mediadora en la relación entre discurso, cognición y sociedad se convierte, de este modo, en imprescindible para entender los límites de la manipulación en las traducciones de noticias que se publican en la prensa.

Una noticia traducida implica una recontextualización obligatoria centrada en el destinatario

\footnotetext{
5 "Cuando los hechos se difunden mediante noticias, se adaptan su forma y sentido de acuerdo con las convenciones propias del género de las noticias" (Trad. propia).
} 
del mensaje, pues supone adaptar el contenido de la misma a un público que no tiene por qué estar familiarizado con la cultura de la LO. El problema radica cuando dicha manipulación del original es ideológica.

Recientemente, algunos estudios de traductología han analizado hasta qué punto la manipulación ideológica en traducción puede afectar el comportamiento del destinatario meta. Uno de estos estudios es el de Bennoui y Kellou (2020), quienes coligen que

[...] the translation receiver is subject to various studies that are undertaken for the purpose of controlling their behavior and reactions with words, $[\ldots]$, emphasizing the power of language considering translation a double-edged weapon when dealt with media field (p. 18). ${ }^{6}$

Para analizar acertadamente la traducción del TO, será preciso considerar también las cuestiones que afectan a nivel macro y superestructural el TM, lo que quiere decir que no puede llevarse a cabo un análisis sobre manipulación en noticias si solo se tiene en cuenta el nivel microestrucual de la noticia per se. La inclusión de la sociedad en el acercamiento metodológico al texto analizado (procedimiento cognoscitivo) será fundamental para interpretar el mensaje en LM, y para asociar, igualmente, las decisiones traductológicas adoptadas por el traductor.

\section{Metodología de trabajo}

En el presente artículo no se estudia la manipulación o demagogia política, en el sentido de analizar las marcas ideológicas que definen a cada diario en concreto, sino que se inves-

6“[...] el destinatario de la traducción es objeto de varias investigaciones que se desarrollan con el propósito de controlar sus comportamientos y sus reacciones con palabras, [...], poniendo énfasis en el poder del lenguaje que considera la traducción como un arma de doble filo cuando se utiliza en el entorno de los medios de comunicación" (Trad. propia). tiga si existen o no marcas de manipulación lingüística incorporadas cuando se traduce el mensaje del to hacia la LM. Aunque nos gustaría poder analizar la noticia completa que mostramos en este trabajo, las constricciones espaciales nos han delimitado bastante, por lo que se han seleccionado algunos fragmentos de la noticia y, en concreto, los correspondientes a los primeros párrafos de esta.

En todo momento se ha tenido en cuenta la información del TO y se ha comparado el enunciado equivalente en el TM, a partir de criterios traductológicos (Hurtado, 2001). En concreto, nos hemos guiado por un acercamiento que comprende las técnicas de traducción general de Hurtado (2001), tales como la generalización, la simplificación, la ampliación lingüística, la modulación, el cambio de perspectiva, la transposición, la omisión, etc.

También nos apoyamos en Abdi (2019), que sobre la base de Schjoldager (2008), señala algunas técnicas traductológicas que miden el nivel de manipulación lingüística que puede llevar a cabo el traductor que trabaja en contextos políticos (según si están dirigidas al то o al тM), y propone un estudio de caso centrado en decretos presidenciales. Algunas de las estrategias comprenden la traducción directa, el calco, la traducción oblicua, la paráfrasis, la adaptación, la condensación lingüística, etc. (Abdi, 2019, p. 886). Debido a las limitaciones espaciales, no ha sido posible incluir un ejemplo de cada técnica en este trabajo.

Además, para valorar los cambios de manipulación hallados entre el To y el TM, nos hemos decantado por el uso del ACD y del triángulo ideológico de Van Dijk (1998), desde el cual se explica la relación entre el discurso y la sociedad por medio de una capa que actúa de mediadora, que es la cognición: el discurso es la parte más concreta (material) del proceso de comunicación, y se matiza por medio de los diferentes mecanismos lingüísticos de la lengua. La cognición, conformada por nuestros conocimientos 
del mundo y las experiencias, nos ayuda a establecer el vínculo entre la sociedad con la que se interactúa y la lengua. Por último, la sociedad precisa de la comunicación para desarrollarse como sociedad (algo que se relaciona con la concepción aristotélica del ser humano como ser social por naturaleza), pero no puede desarrollar la comunicación si primero no asume unas premisas concretas de la lengua para poder favorecer dicho intercambio de comunicación (el discurso) y si no tiene antes un conjunto de conocimientos del mundo comunes con su interlocutor (la cognición). ${ }^{7}$

Así define Van Dijk el concepto asociado al triángulo ideológico discurso/cognición/ sociedad: ${ }^{8}$

7 Este triángulo se relaciona, a su vez, con el paradigma de lengua y habla de Saussure (1916), y de competencia y actuación de Chomsky (1988), en tanto se refiere al intercambio comunicativo (discurso) en una sociedad que comparte unas reglas lingüísticas con las que llevar a cabo el acto de comunicación.

8 "Como se puede deducir de las anteriores observaciones, el marco para un estudio adecuado del discurso se puede resumir en tres conceptos principales, a saber, discurso, cognición y sociedad. Para enfatizar las diversas interrelaciones, podemos ver esos conceptos como si representaran los tres vértices de un triángulo, en el que encontramos relaciones entre discurso y cognición, así como entre discurso y sociedad, mientras que lo mismo es verdad para los otros dos conceptos. Nótese que esas tres nociones se entienden en un sentido amplio. Es decir, 'discurso' aquí implica también lenguaje, uso lingüístico, interacción verbal y comunicación. Del mismo modo, 'cognición' representa tanto la cognición social como la individual, tanto el pensamiento como la emoción, tanto las representaciones de la memoria como los procesos mentales. Y 'sociedad' aquí se entiende tanto en el micronivel de las situaciones y las interacciones sociales como en el macronivel de los grupos, de las relaciones grupales, de las instituciones, de los sistemas abstractos y del orden social en general. En realidad, también la política y la cultura se tornan aquí como propiedades de la sociedad. Puesto que los triángulos suelen representarse sobre dos pies, haremos nosotros lo mismo: su base consiste en el discurso y la sociedad, obviamente li-
Within the broader framework of critical discourse studies my sociocognitive approach to discourse is characterized by the Discourse-Cognition-Society triangle.

Whereas all approaches in cds study the relations between discourse and society, a sociocognitive approach claims that such relations are cognitively mediated. Discourse structures and social structures are of a different nature, and can only be related through the mental representations of language users as individuals and as social members.

Thus, social interaction, social situations and social structures can only influence text and talk through people's interpretations of such social environments.

And conversely, discourse can only influence social interaction and social structures through the same cognitive interface of mental models, knowledge, attitudes and ideologies.9 (2009, p. 64)

gados entre sí por la forma que toma el discurso en situaciones sociales, a saber, como interacción social. Es decir, de izquierda a derecha, la línea de la base del triángulo va desde la microestructura del habla y del texto tomados como interacción social, hasta la contextualización social y las funciones del uso lingüístico, lo que implica también a los participantes en el discurso como actores sociales y los contextos, las localizaciones y estructuras sociales. En este sentido, el triángulo efectivamente debe verse como algo con base y con raíces en la sociedad, que adquiere su relevancia empírica gracias a las actividades relevantes de los actores sociales. [...] En ese sentido es en el que la 'cima' cognitiva del triángulo desarrolla realmente un papel mediador, supervisor, y representa un nivel o una dimensión de un marco conceptual en el que todo literalmente tiene sentido para los participantes" (Van Dijk, 1997, pp. 67-68).

9 "Dentro del amplio marco de los estudios críticos del discurso, mi enfoque sociocognitivo en el discurso se caracteriza por el triángulo discurso/cognición/ sociedad.

A pesar de que en todas las aproximaciones en los estudios críticos del discurso (ECD) se estudian las relaciones entre el discurso y la sociedad, gracias al enfoque sociocognitivo se afirma que dichas relaciones están mediadas cognitivamente. Las estructuras discursivas y sociales son de naturaleza diferente, y 
Mediante el triángulo se revela la aproximación que hace el autor en el análisis del discurso y los elementos que examina para estudiar la ideología. Considera que al unir la dimensión cognitiva y social del triángulo se puede definir el contexto local y global del discurso.

Los objetivos de estudio orientados a la perspectiva del ACD garantizan la inclusión del binomio texto/contexto como componentes esenciales para comprender la ideología que marca las decisiones lingüísticas del acto comunicativo, por medio de las cuales la sociedad produce e interpreta un mensaje en el acto de comunicación del discurso.

Para cumplir las pretensiones del ACD, Van Dijk (2003) afirma que "un adecuado análisis del discurso requiere simultáneamente un detallado análisis cognitivo y social, y viceversa, y que sólo la integración de estas explicaciones puede lograr una adecuación descriptiva, explicativa y, sobre todo, crítica [...]" (p. 147).

El triángulo, por otra parte, supone un marco de estudio adecuado del discurso para enfatizar las diversas interrelaciones entre los tres conceptos que lo conforman. El discurso y la sociedad se unen en el sentido de que todo acto comunicativo es un acto social, fruto de la interacción, y de ahí que esta relación vaya desde la microestructura hasta la contextualización social, lo que sugiere que los interlocutores son actores sociales dentro de un contexto y de una estructura social determinada.

tan solo pueden relacionarse a través de las representaciones mentales de los hablantes como miembros individuales y como miembros sociales.

Por lo tanto, la interacción social, las situaciones sociales y las estructuras sociales tan solo pueden influir en el texto y la conversación mediante las interpretaciones de los hablantes de dichos entornos sociales.

$\mathrm{Y}$ al contrario, el discurso tan solo puede influir en la interacción social y en las estructuras sociales mediante la misma interfaz cognitiva de los modelos mentales, el conocimiento, las actitudes y las ideologías" [Trad. propia].
La cognición representa la cima del triángulo y actúa como mediadora entre la sociedad y el discurso en tanto en cuanto consigue unir a los otros dos conceptos mediante las actividades, procesos y representaciones mentales.

Ninguna descripción de las situaciones sociales o de las estructuras sociales, por una parte, o de las estructuras discursivas, por otra, es completa sin la miríada de los aspectos mentales que han de hacerse explícitos en el plano cognitivo: significado, funciones, comprensión, intenciones e intencionalidad, conocimiento y muchos otros aspectos del discurso entendido como acción e interacción en la sociedad tienen que explicarse en este nivel de la cima (Van Dijk, 1997, p. 68).

Aplicado a nuestro artículo, en el nivel macrotextual del discurso (en nuestro caso, de la noticia que analizamos) se encuentra la sociedad, a la que se le asocian las relaciones de poder, los interlocutores y las estructuras sociales. Van Dijk (1998) concibe el poder social como un medio para controlar la mente y la acción de los grupos sociales. El discurso, por su parte, se hallaría en el nivel microtextual, y se refiere a la lengua y a sus estructuras, gracias a las cuales podemos determinar la ideología.

Así, el triángulo ideológico se concibe como un marco teorético mediante el cual se pueden analizar tanto la comprensión como la producción textuales, siempre unidas al contexto dado y a la sociedad. Al respecto, nuestro estudio se dirige a analizar una traducción que ha de contemplar varios aspectos en el TM: la sociedad (lectores en LO) a la que va dirigida el To (discurso), teniendo en cuenta que la narración de la noticia ha de englobarse dentro de la forma de ver el mundo que concibe el periódico (ideología), razón por la que ciertos lectores prefieren leer dicho periódico y no otro, con otra ideología diferente.

En el siguiente apartado analizamos la noticia que hemos indicado anteriormente, seleccionada al azar dentro de las 56 que conforman nuestro corpus para mostrar el modelo de análisis. E1 
Español (1)

LA habANA - R.P. es un "emprendedor" cubano. Su pequeña empresa reduce su estructura a él y su automóvil, que no son ni una persona ni un automóvil cualquiera. R.P. tiene cuarenta años y hace dieciséis se graduó como ingeniero mecánico en una universidad tecnológica habanera, pero desde hace seis, al calor de la política de ampliación del trabajo privado, dejó su antigua labor y se dedicó a conducir autos de alquiler. Casi de un día para otro su salario aumentó en cinco, seis veces. Y su auto, tan peculiar y "cubano" como él, es un Chevrolet Bel Air de 1957 que heredó de su padre... (Padura, 2017a).

contexto de la misma es determinante para poder entender algunas decisiones traductológicas que parecen haberse visto influenciadas por factores dependientes de las relaciones diplomáticas de los dos países sobre los que gira la noticia, esto es, Estados Unidos y Cuba, por lo que nos servirá como un ejemplo muy representativo para llevar a cabo nuestro análisis derivado del ACD.

\section{Análisis traductológico de una noticia del periódico The New York Times}

En este apartado analizamos tres fragmentos de la noticia que nos ocupa. A continuación se presenta el Fragmento 1.

Como observamos en (2), ${ }^{10}$ la primera oración que aparece afirma el hecho de que el protagonista de la historia (R.P.) es un emprendedor de la ciudad de Cuba. Sin embargo, si atendemos a la versión original, el término "emprendedor" aparece entre comillas.

10 En el texto, las referencias numéricas entre paréntesis del tipo (1), (2), (3), etc., aluden a las versiones en inglés o español de cada fragmento. Así, toda referencia a (1), por ejemplo, se refiere al extracto ofrecido en el fragmento 1 en la versión en español, y el número (2), a la versión en inglés del fragmento 1 . Estas asociaciones son similares para (3) y (4) (ES e EN, respectivamente) para el fragmento 2 y para (5) y (6) para el fragmento 3.
Inglés (2)

HAVANA - R.P. is a Cuban entrepreneur. His business consists of him and his car - but this is not just any man and any car. R.P. is 40 years old. Sixteen years ago he graduated with a degree in mechanical engineering from Havana's Technological University, but six years ago, when government relaxed the rules on private enterprise, he left his transportation specialist job to become a taxi driver. Almost immediately he started earning five, six times his previous salary. His car, of course, is as idiosyncratic and Cuban as he is: a 1957 Chevrolet Bel Air he inherited from his father. (Padura, 2017b).

A priori, podemos entender que el término entrecomillado se refiere a algún uso connotativo especial otorgado por el autor, razón por la que se ha empleado tal signo ortográfico. Como nos recuerda el Diccionario de la lengua española de la Real Academia Española (RAE), la Fundéu y las principales guías de estilo, las comillas se utilizan, entre otros usos, para señalar el carácter especial que adquiere la significación de una palabra, justificación entendible, en los contextos periodísticos, dentro de los géneros de opinión. No obstante, cabría interpretar el uso de las comillas aquí para designar una categoría existente en el sistema laboral cubano (por ejemplo, en el sistema cuentapropista de Cuba, el "emprendedor" puede ser una categoría profesional oficial, en cuyo caso serían válidas las comillas, siempre que se refiera a la denominación específica de tal profesión en un país en concreto). La cuestión, sin embargo, puede responder a diferentes lecturas, ${ }^{11}$ aten-

11 Se ha consultado a varios expertos sobre esta cuestión (la consulta a expertos forma parte, también, del proceso documental en traducción, especialmente cuando se trata de un reto traductológico tan delicado como este). Algunos de ellos se han decantado por la primera posibilidad; otros, por la segunda. Un tercer grupo admitía las dos posibilidades como válidas dependiendo de cuál es la intencionalidad del autor del original. El traductor tiene la obligación de consultar fuentes fidedignas antes de optar por una u otra decisión, ya que, como estamos comprobando mediante este ejemplo, cuando se trabaja con textos 
diendo a cuál de estas dos posibilidades nos convence más para explicar el uso de las comillas (uso descriptivo o aclaratorio, por un lado, o irónico/satírico para señalar un significado especial atribuido a dicho término, por otro). Ante esta disyuntiva tan delicada, el traductor debe consultar al autor del original para aclarar este interrogante, siempre que le sea posible. En este caso, la versión en inglés opta por eliminar las comillas directamente.

A continuación, la información que ofrece la versión en español, mediante una única oración, se amplía en dos en la versión en inglés:

(1) R.P. tiene cuarenta años y hace dieciséis se graduó como ingeniero mecánico en una universidad tecnológica habanera, pero desde hace seis, al calor de la política de ampliación del trabajo privado, dejó su antigua labor y se dedicó a conducir autos de alquiler (Padura, 2017a).

(2) R.P. is 40 years old. Sixteen years ago he graduated with a degree in mechanical engineering from Havana's technological university, but six years ago, when government relaxed the rules on private enterprise, he left his transportation specialist job to become a taxi driver (Padura, 2017b).

Se observan diferentes modulaciones o cambios de perspectiva (Hurtado, 2001): por ejemplo, en español se hace referencia al resultado de esos estudios ("Graduarse como ingeniero mecánico"), mientras que en inglés se señala el título universitario en concreto (Mechanical engineering). La universidad queda perfectamente explicitada en la versión en inglés (Havana's Technological University), pero si se observa detenidamente, en el original se generaliza dicha universidad y simplemente se dice que es una "universidad tecnológica habanera".

\footnotetext{
"sensibles" (Simms, 1997) como los de naturaleza política (con posibles marcas ideológicas), el traductor ha de buscar la objetividad en el trasvase hacia la LM para garantizar la fidelidad y la deontología ética en el ejercicio de nuestra profesión.
}

Posteriormente, observamos en la versión en español el enunciado "al calor de la política de ampliación del trabajo privado", al que le corresponde la oración en inglés "government relaxed the rules on private enterprise". E1 falso sentido (Abdi, 2019) es evidente: en (2), simplemente se indica que el Gobierno "relajó" las políticas que se cernían sobre la empresa privada. En (1), con un claro componente literario y retórico, realza "la ampliación del trabajo privado". En este caso, el término "calor" funciona como sinónimo de "al amparo de la política de ampliación". Precisamente, cualquier política de ampliación trata de un acercamiento más escrupuloso al tema del que se trate, motivo por el que es difícil concebir la realidad presentada en (2) por medio de ese participio "relaxed".

El fragmento continúa con "he left his transportation specialist job to become a taxi driver", lo que se corresponde en (1) con "dejó su antigua labor y se dedicó a conducir autos de alquiler". Es cierto que, en ambos enunciados, se pone de manifiesto que el protagonista (R.P.) ya no trabaja más en su negocio de siempre, pero en (2) se ha añadido una información que en (1) no se pone de manifiesto: "dejó su antigua labor" señala, simplemente, el hecho de que abandonó su viejo oficio; sin embargo, en (2) se añade el matiz correspondiente de dicho trabajo. Esta especificación, como se comprueba, no aparece como tal en la versión en inglés: "he left his transportation specialist job". Además, el empleo por el que sustituye su antiguo oficio aparece señalado en (1) como "conductor de autos de alquiler", lo que en (2) se simplifica a "taxi driver". No obstante, pragmáticamente, no supone lo mismo trabajar como "taxista" que como "conductor de autos de alquiler".

La noticia continúa con la afirmación "Casi de un día para otro su salario aumentó en cinco, seis veces", cuyo equivalente en (2) es "Almost immediately he started earning five, six times his previous salary". Si bien es evidente la modulación sintáctica y lingüística que se ha llevado a cabo al desarrollar el proceso de traducción, 
la equivalencia podría asociarse sin mayores problemas, desde un punto de vista semántico.

Para finalizar con el análisis de este fragmento, nos centramos ahora en el último enunciado: "Y su auto, tan peculiar y 'cubano' como él, es un Chevrolet Bel Air de 1957 que heredó de su padre...", que se corresponde en (2) con "His car, of course, is as idiosyncratic and Cuban as he is: a 1957 Chevrolet Bel Air he inherited from his father". El uso de las comillas para señalar el término "cubano" hace referencia al uso especial (e irónico) que sugiere el autor del To al expresar que el coche típicamente cubano sea fabricado en Estados Unidos. Por su parte, no existe correspondencia en la versión en inglés, la cual elide el uso de esas comillas, de modo que no se puede apreciar ese valor connotativo que ha empleado el autor en el to desde el punto de vista pragmático. Se trata de una paradoja simbolizada en el "auto" y que remite al contexto político existente entre ambos países en la actualidad. ${ }^{12}$ También es curiosa la traducción del término "peculiar" por "idiosincrático", cuando las opciones de traducción literal son bastante abundantes en inglés: strange, odd, peculiar, etc., según el sentido.

Desde el punto de vista del ACD, debemos tener en cuenta que el autor, Leonardo Padura, periodista cubano de alto reconocimiento en la prensa, se dirige a la sociedad que representa el destinatario para quien va dirigido el texto informativo, esto es, los lectores del The New

12 No obstante, y en consonancia con la nota anterior, cabe decir que algunos de los expertos a los que se les ha consultado también han admitido la posibilidad de interpretar el uso de estas comillas como irónicas (como una burla, al afirmar que el auto es tan cubano como él). De nuevo, la consulta a expertos se determina como un factor fundamental antes de tomar cualquier decisión traductológica, con el fin de considerar diferentes puntos de vista, especialmente en este tipo de contextos, donde se comprueba, además, que un signo ortográfico puede llegar a ser muy determinante en cuanto a la traducción.
York Times. Tengamos en cuenta también que las distintas connotaciones apreciadas en el то han de entenderse dentro del prisma ideológico que pone de manifiesto el medio en que se publica la noticia, es decir, el propio periódico, que propicia la relación entre la sociedad y el mismo mensaje de la noticia, el discurso, mediante esa capa mediadora que sería la cognición. Esta está ligada, en este fragmento, a los conocimientos culturales que se le presuponen al lector de la noticia (por ejemplo, interpretar adecuadamente la paradoja del automóvil).

Los conocimientos culturales y especialmente históricos y políticos son imprescindibles en este fragmento, pues suponen la mediación necesaria para interpretar el sentido del то. Las faltas de correspondencia, no obstante, no eximen de responsabilidad al traductor, si bien debemos considerar que a partir del to no se cuenta con los datos suficientes para saber si las modificaciones las ha llevado a cabo el traductor o si responden a criterios de simplificación y acortamiento prototípicos de las normas editoriales sobre noticias traducidas en prensa, como bien explican Rovena y Marchan (2017).

En segundo lugar, nos ocupamos del Fragmento 2, sobre el que llamamos especialmente la atención por el interés que denota desde el punto de vista traductológico.

Si atendemos a la distribución visual, la longitud del fragmento muestra que hay mucha información que ha sido suprimida en la versión en inglés. El primer enunciado comienza del modo siguiente en (3):

Hace un año, ante el auge de visitantes estadounidenses (nunca turistas, los estadounidenses no viajan a Cuba como turistas pues la ley de embargo se los prohíbe) [...], R.P. tomó la decisión que ya otros de sus colegas habían adoptado: convertiría su Chevrolet sedán en un descapotable, el más cotizado de los transportes en que esos visitantes gustan de recorrer La Habana, no importa lo implacable que sea el sol del trópico (Padura, 2017). 
Fragmento 2

Español (3)

Hace un año, ante el auge de visitantes estadounidenses (nunca turistas, los estadounidenses no viajan a Cuba como turistas pues la ley de embargo se los prohíbe), R.P. decidió reorientar su negocio: en lugar de recorrer La Habana buscando pasajeros que le pagaran en moneda nacional, se especializaría en pasear visitantes estadounidenses. Porque a los vecinos del norte les encanta recorrer la mítica, magnética ciudad de La Habana en los mismos autos que más de medio siglo atrás pudieron haber usado sus abuelos en sus viajes a Cuba. Y pagan bien por ese capricho. Para que el negocio funcionara mejor, R.P. tomó la decisión que ya otros de sus colegas habían adoptado: convertiría su Chevrolet sedán en un descapotable, el más cotizado de los transportes en que esos visitantes gustan de recorrer La Habana, no importa lo implacable que sea el sol del trópico. Para hacer la "cirugía reconstructiva" de su auto, R.P., que prefiere no usar su nombre completo para evitar ver afectado su negocio, buscó a otros emprendedores que se han especializado en este tipo de transformación e invirtió en ella todos sus ahorros y más: 3000 dólares.

Ahora, ante las amenazas del presidente Donald Trump de revisar la política de acercamiento entre Cuba y Estados Unidos, R.P. no sabe si el "emprendimiento" que creía próspero seguirá siéndolo o si ha hecho la peor inversión de su vida porque, sin afluencia de clientes, demorará años en amortizarse... R.P. ruega porque Trump no le joda el negocio. (Padura, 2017a).
Inglés (4)

A year ago, R.P. converted his sedan into a convertible, the mode of transportation most sought-after by the new crowds of American visitors keen to take in Havana's sights. To perform this reconstructive surgery, R.P. spent his entire savings and more, some $\$ 3,000$. But in November, the American president-elect, Donald J. Trump, threatened to roll back the countries' newly restored relations. Now R.P. has no idea whether his business will continue to prosper or if he just made the worst investment of his life. (Padura, 2017b).
Hemos seleccionado únicamente la información que tiene correspondencia entre ambas versiones. Al fragmento seleccionado le corresponde el enunciado siguiente en (4):

A year ago, R.P. converted his sedan into a convertible, the mode of transportation most sought-after by the new crowds of American visitors keen to take in Havana's sights ( $\mathrm{Pa}$ dura, 2017).

En inglés, se ha omitido prácticamente toda la proposición destinada a señalar una aclaración que introduce el autor del texto original, la cual incluye determinados matices ideológicos ("ante el auge de visitantes estadounidenses nunca turistas, los estadounidenses no viajan a Cuba como turistas pues la ley de embargo se los prohíbe-") que, en vistas del triángulo ideológico de Van Dijk (1998), supondría el hecho de suprimir aspectos de la sociedad que se adquieren mediante la cognición en la versión original (como la omisión de expresiones de carácter ideológico) y que, de algún modo, aparecen degradados o bloqueados (es decir, omitidos) en la versión meta, esto es, la versión en inglés.

Parece que la aclaración sobre la terminología que ha de emplearse con los turistas procedentes de Estados Unidos (la diferencia entre "turista" y "visitante") no precisa ser especificada en la versión en inglés, y la aclaración se omite, pues da por hecho que el lector del TM interpretará el mensaje según la cognición del saber común ligado a la sociedad estadounidense.

Otra alternativa sería incluir la orientación ideológica del The New York Times y la opinión que la dirección editorial del periódico tiene 
con respecto al asunto tratado (turistas y visitantes) que, no obstante, no parece oportuno analizar, puesto que no es una información a la que pueda accederse mediante el texto analizado (el discurso), aunque es interesante a efectos de la cognición y la interpretación del mensaje de la sociedad del TM.

Posteriormente, en (3) se señala que "R. P. tomó la decisión que ya otros de sus colegas habían adoptado: convertiría su Chevrolet sedán en un descapotable", mientras que en (4) se indica que " $R$. P. converted his sedan into a convertible". La información que aparece en (4) resume muy sucintamente los datos que sí aporta (3), reduciendo el matiz literario e informativo de la historia a un breve sumario.

En (3) se nos informa que la decisión que tomó R. P. no era excepcional, pues ya ha habido otras personas que lo habían hecho con anterioridad (información contextualizada dentro de la cognición de la cultura origen). Por su parte, en (4) se prescinde de esta información, como si no fuera significativa para la historia (omisiones dentro del discurso meta que configuran el modo de interpretar el mensaje a través de la cognición de la cultura meta).

Tengamos en cuenta que en el párrafo inicial se explicita claramente que R.P. (y su automóvil) "no son ni una persona ni un automóvil cualquiera" (alusión a la paradoja sobre el automóvil a la que apuntábamos anteriormente). Sin embargo, los detalles que justifican este tipo de aseveraciones no aparecen tan puntualizados en la versión en inglés.

El matiz literario que se advierte al introducir el condicional "convertiría" (un futuro visto desde el pasado) se elimina en la versión meta y se reemplaza por un pasado simple del modo indicativo, que expresa datos relativos a la realidad en un momento ya acontecido. Por tanto, afirma el hecho de forma más directa que en el TO, un modo de articulación del discurso acorde con el resto de las decisiones traductológicas llevadas a cabo en el TM, que implican, de cierta manera, el modo de dirigirse al público meta que adopta el discurso (la noticia) en inglés para su interpretación.

Siguiendo con la dinámica del acortamiento, elimina también el término "Chevrolet", el cual, aunque queda probablemente especificado gracias al contexto (de nuevo, cognición), resulta fundamental en la totalidad de la noticia, pues recordemos que este es uno de los términos que componen el título de la noticia en español y en inglés ("Chevy" es el modo coloquial con el que se apoda a los coches de la marca Chevrolet en Estados Unidos).

En (3), el siguiente enunciado incluye el término "cirugía reconstructiva" entre comillas, para señalar un significado especial que se le atribuye en este caso a dicho término. En inglés, se da por sentado que se interpretará adecuadamente esta metáfora, motivo por el que se omiten las comillas (otro efecto traductológico que puede entenderse dentro de la cognición asociada a la cultura meta). No obstante, esta colocación no casa de forma adecuada con el contexto de la versión en inglés: en (4) se incluye el demostrativo "this", que concreta una información que en apariencia es conocida por el lector. Sin embargo, no se ha usado este término con anterioridad, por lo que, de alguna manera, esta información resulta extraña para el lector en LM.

Además, tampoco se indica sobre qué se llevará a cabo la "cirugía reconstructiva", como sí lo especifica la versión en español ("su coche"). Las simplificaciones y omisiones del TM, por ende, repercuten en la calidad del mensaje traducido, y también influyen en el contenido intrínseco del mensaje y en los distintos matices que ayudan a interpretar la información desde un punto de vista sociocognitivo (Van Dijk, 1996).

La siguiente información detallada en el fragmento (3) tiene muy pocas correspondencias reales con lo enunciado en (4). En el texto en inglés no se indica, en ningún momento, el deseo de R.P. de que no se utilice su nombre completo, motivo por el que se usa en todo momento una sigla: "prefiere no usar su nombre 
completo". El motivo viene también especificado: R.P. teme que, si le reconocen, pueda ser que su negocio se venga abajo ("para evitar ver afectado su negocio"). También se omite la información sobre que R.P. ha consultado a otras personas que antes que él realizaron un cambio de infraestructura tan grande como al que él se enfrenta ahora. Esta decisión, lejos de justificar la omisión, tiene coherencia en (4), dado que antes también ha omitido la información relativa a que él no ha sido el único en realizar este cambio ("la decisión que ya otros de sus colegas habían adoptado").

En lugar de traducir el verbo "invertir" en inglés, se ha utilizado el verbo "spent". Tómese en consideración que "invertir" se define, en el Diccionario de la lengua española de la RAE (2020) como "Emplear, colocar un caudal" (económico, generalmente), y que, por su parte, spend, cuya traducción habitual en español es "gastar", "malgastar" o incluso "desperdiciar", se refiere a "consumir", "destruir" o "emplear el dinero en algo".

Observemos, además, que la correspondencia en un diccionario monolingüe en inglés es prácticamente similar: el diccionario $\mathrm{Me}$ rriam-Webster (2020) traduce el verbo spend por "To consume wastefully", e invest, traducción habitual de "invertir", por "To commit [money] in order to earn a financial return". Por tanto, en este caso se altera ligeramente la información detallada entre las dos versiones.

Aunque el matiz es mínimo, también es importante indicar la diferencia entre afirmar rotundamente que lo que se gastó fueron, en concreto, "3000 dólares", frente a "some $\$ 3,000 "$. En este sentido, la afirmación no adquiere la misma fuerza ilocutiva, siguiendo la terminología de Anscombre y Ducrot (1983): no es lo mismo afirmar que se gastó una cantidad en concreto (lo que transmite seguridad en la aseveración), que decir, en términos de probabilidad, que se gastaron, "más o menos", "unos tres mil dólares". Estos cambios traductológicos, en esta ocasión, no responden a criterios relacionados con la cultura o la cognición, ya que se trabaja directamente desde el plano microtextual del discurso ofrecido. Así pues, las modificaciones se llevan a cabo desde el discurso mismo, por lo que estos cambios en la configuración del mensaje no pueden entenderse, a efectos del ACD, desde un punto de vista sociocognitivo justificable.

Antes de abordar el siguiente enunciado, que sí presenta correspondencia con la versión original, fijémonos en este breve fragmento, ampliado en la versión en inglés:

To perform this reconstructive surgery, R.P. spent his entire savings and more, some $\$ 3,000$. But in November, the American president-elect, Donald J. Trump, threatened to roll back the countries' newly restored relations (Padura, 2017b).

Llama la atención que se introduzca un enunciado que no procede de la traducción directa del то. Es la primera vez en la noticia en inglés que se localiza una información añadida, lo que se conoce como "adición injustificada" (Abdi, 2019), también desde el punto de vista interpretativo de la cognición. Esto se entendería, desde la teoría del ACD, como un modo de representación negativa del Otro (Van Dijk, 1998), ya que se está promoviendo una representación que intensifica una información ideológica con sesgos políticos en el TM, que no aparecen previamente en el TO.

En esta explicitación, se incluye una fecha concreta (el mes de noviembre), se indica la persona que efectúa la acción ("el presidente estadounidense" y, mediante una aposición, se especifica el nombre: Donald J. Trump), que representa al actante, y, por último, se indica la acción: "threatened to roll back the countries' newly restored relations", lo que podría traducirse de manera literal por "amenazó con hacer que las relaciones que se habían vuelto a establecer entre los países retrocedieran".

La información semántica contenida en el verbo "threaten" ("amenazar", en español), confirma 
un determinado sesgo ideológico, ya que implica una información diferente que la que pudiera transmitir un verbo dicendi similar, pero mucho más neutro: "decir". Las implicaciones semánticas, relativas en concreto a la fuerza pragmáti$\mathrm{ca}$, son diferentes, puesto que se añaden valores que no se incluyen en la versión original.

E1 fragmento continúa en (3) con:

(3) Ahora, ante las amenazas del presidente Donald Trump de revisar la política de acercamiento entre Cuba y Estados Unidos, R.P. no sabe si el "emprendimiento" que creía próspero seguirá siéndolo o si ha hecho la peor inversión de su vida porque, sin afluencia de clientes, demorará años en amortizarse... (Padura, 2017a).

Su correspondencia en (4) es la siguiente:

(4) Now R.P. has no idea whether his business will continue to prosper or if he just made the worst investment of his life (Padura, 2017b).

A lo largo del análisis, se observan dificultades para encontrar el equivalente exacto de un enunciado, pues dada la escasa correspondencia formal entre una versión y otra, y los cambios sintácticos que hallamos en la configuración de las oraciones, es difícil proceder a la comparativa de manera rápida. En primer lugar, la versión en español incluye, en este caso, una información relacionada con la que detallaba con anterioridad: "ante las amenazas del presidente Donald Trump de revisar la política de acercamiento entre Cuba y Estados Unidos". Podría tratarse de la correspondencia con el enunciado final que acabamos de comentar, pero, en ese caso, habría que admitir un nuevo cambio, puesto que en la versión en inglés se habla de políticas que "se han restablecido de nuevo", lo que podría entenderse como que en algún momento se rompieron, y ahora habla, en la versión original, simplemente de "política de acercamiento", lo que tampoco tiene una correspondencia directa.

El siguiente enunciado resume sobremanera la información contenida en (3): "R.P. no sabe si el 'emprendimiento' que creía próspero seguirá siéndolo o si ha hecho la peor inversión de su vida". Ahora, esta información queda resumida, en (4), así: "Now R.P. has no idea whether his business will continue to prosper or if he just made the worst investment of his life". El término "emprendimiento" aparece en español entre comillas, algo que nos recuerda al modo de referirse al negocio del protagonista que hizo el autor del to al principio del texto cuando hablaba de "emprendedor" (entre comillas). Este rasgo connotativo se ha vuelto a elidir en la versión traducida, de modo que el lector en lengua inglesa no es capaz de reconocer la verdadera finalidad connotativa a la que aludía el autor original, cuando la traducción debe proveer al texto de los rasgos suficientes como para garantizar que las mismas impresiones recibidas en el TM sean las que recibe originalmente un lector en el To. Esta propiedad del proceso traductor se estudia en traductología con el concepto acuñado por Nida (1964) de equivalencia dinámica. No obstante, son escasos los enunciados de la noticia a los que se les puede asociar una equivalencia formal y dinámica directa. Desde el punto de vista del ACD, las diferencias de este ejemplo vuelven a estudiarse desde el plano microtextual y no desde el social, ya que no existen relaciones directas por las que la cognición en LM obligue a modificar el enunciado del to. Los cambios se introducen desde el discurso y hacia el discurso mismo (en LM), sin responder a criterios lingüísticos per se.

Ese "Emprendimiento que creía próspero" se resume simplemente por "business" en la versión en inglés, lo que reduce los sesgos connotativos asociados a la versión origen. El fragmento en inglés termina aquí. Sin embargo, el fragmento en español, como vemos en (3), añade otros datos que no aparecen en la versión traducida.

En inglés, se ofrece simplemente la información de que puede que se trate de "la peor inversión de su vida", pero no especifica el porqué, algo que sí aparece en la versión original. Además, elide una oración importante para entender el carácter connotativo que debe serle otorgado al To: "R.P. ruega porque Trump no 
le joda el negocio". Esta proposición es simplemente omitida. Los motivos pueden ser varios, pero está claro que la asociación cognitiva que se le atribuye al to al leer un enunciado como este ayuda a entender la panorámica ideológica del texto en sí, algo que queda muy sutilmente matizado - si acaso- en el texto en inglés (cognición).

El texto en inglés, además de resumir, parece estar reescrito de un modo más "políticamente correcto", y elide en todo momento vocablos que puedan tener connotaciones negativas, en un intento por consolidar una información neutra en la noticia en inglés (decisión probablemente adoptada por la dirección editorial de la versión en inglés del periódico, la cual informa del estilo que han de seguir el traductor y el revisor a la hora de trasladar el contenido a la LM).

Por su parte, en el último enunciado, que señala una proposición desiderativa emitida por el protagonista, marcada con el uso del subjuntivo, se observa un verbo "políticamente incorrecto", que podría proceder o bien de la inventiva narrativa del propio autor para poner énfasis en su artículo (nivel microtextual, donde se hace patente la función poética del lenguaje, a través de la libertad creativa o literaria del autor), o bien de la expresión literal que hubiera indicado R.P. al autor de la noticia en su momento, en cuyo caso se deberían ha- ber empleado las comillas, para señalar que se trata de una información literal.

Sea como fuere, el hecho de que aparezca un término tan coloquial en una noticia llama sumamente la atención. Este carácter coloquial y despectivo, incluso negativo desde el punto de vista connotativo, otorga un conjunto de matices interesantes para entender la ideología presente en el TO, algo que no puede apreciarse de modo similar en la traducción en el TM (una manipulación sobre el to desde la óptica estilística, la cual, como indicábamos anteriormente, puede responder a criterios fijados por las políticas editoriales).

Veamos ahora el Fragmento 3.

En primer lugar, la parte inicial del fragmento 3 en español no ofrece correspondencias con el fragmento análogo en inglés. Hasta la mitad de (5) no empezamos a ver alguna equivalencia más o menos similar a la de (6).

Obsérvese, en segundo lugar, el siguiente enunciado en español:

(5) Mientras en Cuba se celebraba el homenaje póstumo a Fidel Castro al que asistían personalidades políticas de todo el mundo, un cintillo de la transmisión de CNN en Español advertía que el presidente electo de Estados Unidos prometía revisar el curso de las

\section{Fragmento 3}

Español (5)

Inglés (6)

Mientras en Cuba se celebraba el homenaje póstumo a Fidel Castro al que asistían personalidades políticas de todo el mundo, un cintillo de la transmisión de CNN en Español advertía que el presidente electo de Estados Unidos prometía revisar el curso de las relaciones de su país con la isla y quizá revertir el proceso de acercamiento iniciado en diciembre de 2014 por los presidentes Barack Obama y Raúl Castro.

Uno de los signos más visibles de la mejoría de las relaciones fue la ampliación de categorías en que podían inscribirse los estadounidenses que desearan viajar a la isla y el reinicio de vuelos comerciales directos. El más retumbante, la revisión de los privilegios migratorios de los ciudadanos cubanos (Padura, 2017a).

Barack Obama and Cuba's president, Raúl Castro, began the process of loosening the embargo and strengthening diplomatic ties between their two countries in December 2014. One of the most visible signs of these improved relations was the expansion of Americans' ability to visit the island and the reinstatement of commercial flights. The most notable of all was the revocation in January 2017 of the "wet foot, dry foot" policy under which Cubans who reached the United States could stay - a policy that the Cuban government had greatly resented (Padura, 2017b). 
relaciones de su país con la isla y quizá [...] (Padura, 2017a).

Este enunciado aparece sin traducción en inglés. Se localiza alguna semejanza a partir de "revertir".

Fijémonos en la estructuración sintáctica de la traducción: se ha cambiado de orden la estructura sintáctica que aparece en el To, para dar lugar a una traducción libre (Hurtado, 2001). Así, podríamos establecer, de manera esquemática, una correspondencia formal entre ambas versiones del modo siguiente:

A. (5) [...] revertir el proceso de acercamiento iniciado en diciembre de 2014 (Padura, 2017a).

(6) $[\ldots]$ began the process of loosening the embargo and strengthening diplomatic ties between their two countries in December 2014 (Padura, 2017b).

B. (5) [...] por los presidentes Barack Obama y Raúl Castro (Padura, 2017a).

(6) [...] Barack Obama and Cuba's president, Raúl Castro (Padura, 2017b).

C. (5) [...] Uno de los signos más visibles de la mejoría de las relaciones fue la ampliación de categorías en que podían inscribirse los estadounidenses que desearan viajar a la isla y el reinicio de vuelos comerciales directos $(\mathrm{Pa}$ dura, 2017a).

(6) [...] One of the most visible signs of these improved relations was the expansion of Americans' ability to visit the island and the reinstatement of commercial flights (Padura, 2017b).

D. (5) [...] El más retumbante, la revisión de los privilegios migratorios de los ciudadanos cubanos (Padura, 2017a).

(6) $[\ldots]$ The most notable of all was the revocation in January 2017 of the "wet foot, dry foot" policy under which Cubans who reached the United States could stay - a policy that the Cuban government had greatly resented (Padura, 2017b).

Como se observa, el orden sintáctico no se corresponde en ambos casos.

Nos centramos ahora en la información contenida en (6), que se corresponde, aproximadamente, con la información traducida en (5). En el caso A), se descubre una ampliación lingüística, puesto que se detallan datos que no aparecen en el original y que describen el proceso al que hace referencia (5). No obstante, dado el carácter del texto (macro y superestructuras textuales), se da por hecho que el lector estará más o menos familiarizado con la información que se detalla y el contexto en el que se ubica (presuposición basada en la cognición de la cultura relativa a la sociedad, tanto del то como del TM, en cada caso).

Por otra parte, si se decide justificar determinados fragmentos, lo apropiado hubiera sido actuar de manera semejante, mediante la misma técnica de ampliación y descripción, con todos aquellos casos similares en los que se introduce una información de corte político que el traductor considera que debe aclarar al lector meta. La carencia de decisiones homogéneas ante circunstancias traductológicas semejantes repercute en la escasez de coherencia en las decisiones traductológicas adoptadas, algo que puede estudiarse desde un punto de vista más amplio que el del propio triángulo ideológico de Van Dijk (1998): la falta de revisión del TM (dada la rapidez que impera en los contextos periodísticos), la posible inexistencia de un protocolo para el proceso traductor en la agencia, su incumplimiento por parte del traductor o revisor, etc. (motivos comunes en contextos de traducción periodística, como señala Castillo, 2016).

Este proceso de acercamiento se ha descrito en la versión en inglés como "the process of loosening the embargo and strengthening diplomatic ties between their two countries". Utilizando los datos del triángulo ideológico de Van Dijk (1998), se podría justificar la añadidura de información como un intento para formar una representación concreta de la realidad que ayude al destinatario meta a interpretar la realidad a la que se alude en el тo.

En B), se observa una información bastante similar, si bien con una disposición sintáctica diferente: en (5), se utiliza una aposición especificativa 
unida al sustantivo "presidentes". El enunciado queda distribuido dentro del mismo sintagma preposicional formado por la preposición "por" y seguido del sintagma nominal cuyo núcleo es "presidentes". Compruébese que todo este enunciado representa la función de complemento agente del participio "iniciado" de la oración anterior.

Por su parte, en (6) se habla directamente de Barack Obama, del que no se dice literalmente que sea "presidente", ya que se supone que es un rasgo que el receptor meta asociará inmediatamente (relación discurso/cognición/ sociedad), pero sí se especifica, mediante un genitivo sajón, en el caso del presidente de Cuba, Raúl Castro. Mientras que en (5) se generaliza y se indica la condición de presidencia para ambos dirigentes, en (6) se observa una particularización por la que se adaptan las referencias culturales a la situación cognoscitiva y cultural del lector meta.

En C), por otro lado, observamos que se ofrece una información similar, si bien con matices diferenciadores. Las divergencias más notorias se encuentran en "ampliación de categorías en que podían inscribirse los estadounidenses que desearan viajar a la isla" y en su correspondiente "the expansion of Americans' ability to visit the island". En español, se específica en qué consiste la "ampliación", referida a esas "categorías" en las cuales los estadounidenses que quieran visitar la isla podrían comenzar a inscribirse. En inglés, se reduce la información por medio de una simplificación lingüística y se queda únicamente con el mensaje más importante de todo el enunciado: referir la posibilidad de una expansión mediante la que los estadounidenses puedan visitar la isla.

En cuanto al enunciado en (5) de "el reinicio de vuelos comerciales directos" y su correspondiente (6) "the reinstatement of commercial flights", no se aprecian apenas diferencias semánticas.

Finalmente, en lo que respecta al apartado D), se aprecia una ampliación lingüística con descripción en la versión en inglés. La información de (6) es mucho mayor que en (5). Las correspondencias más básicas serían las de "El más retumbante", traducido por "The most notable of all" y "la revisión de los privilegios migratorios de los ciudadanos cubanos" por: "the revocation in January 2017 of the wet foot, dry foot policy under which Cubans who reached the United States could stay - a policy that the Cuban government had greatly resented".

Los "privilegios", a los que se refiere el то, quedan matizados y explicados en el TM, empleando para ello los términos habituales con los que la prensa se refiere a dichos "privilegios". Como puede apreciarse, en la versión del TM se cuenta con información contextual (“January 2017'), asociada al conocimiento del mundo del que parte el то, que al tiempo se le presupone al TM, y que es una información cultural a la que únicamente se puede acceder mediante una extensa documentación muy bien asentada en el marco de cognición política en el que se inserta esta noticia (the "wet foot, dry foot" policy), además de otros datos informativos que añade el traductor en inglés (" a policy that the Cuban government had greatly resented"). La interpretación de ese contenido interpretativo se asocia en el TM por medio de la cognición del discurso ligado a la sociedad meta.

Estas líneas nos permiten constatar que si bien para traducir el TM se ha tenido presente la información y el boceto del To, es evidente la reformulación —en varios sentidos-que se ha llevado a cabo en la versión en inglés, que lejos de promover la correspondencia semántica idéntica entre ambas versiones (la equivalencia dinámica que nombrábamos con anterioridad), ha optado, más bien, por la utilización de distintas estrategias más propias de la reformulación.

\section{Conclusiones}

Los ejemplos analizados nos han permitido acercarnos a la traducción de una noticia en 
prensa desde un enfoque teórico y metodológico que se ha decantado por la inclusión de las categorías propias del triángulo ideológico del ACD de Van Dijk (1998). De esta manera, se ha analizado la noticia traducida hacia el inglés no solo desde el punto de vista de la traducción, sino también desde el punto de vista pragmático y discursivo, atendiendo, en concreto, a los tres conceptos que conforman el triángulo: 1) la sociedad, a la que nos hemos dirigido como el destinatario final de la traducción; 2) el discurso, que en este caso ha simbolizado la propia noticia, desde el nivel microtextual; y 3) la capa mediadora entre la sociedad y el discurso, que es la cognición (ideología). Como se ha visto, la cognición está ligada a los conocimientos del mundo, de la cultura y de la sociedad, que delimitan tanto el mensaje del To como el del TM, pues muchas decisiones traductológicas dependen en exclusiva de las consideraciones a las que llega el traductor desde la perspectiva sociocognoscitiva.

Se han analizado un total de tres fragmentos diferentes, con versión en español e inglés. Muchos de los enunciados analizados han mostrado que no existía correspondencia directa entre el To y el TM en lo que se refiere al sentido. Si bien es cierto que algunos de esos enunciados se engloban dentro de la sensibilidad asociada al género político, dada la ideología inherente al mismo, no todos los argumentos que pueden asociarse a las decisiones traductológicas pasan por un ejercicio de manipulación entendido en términos ideológicos. Por ello, no ha de asumirse que todo giro en el texto es una manipulación deliberada orientada por lo ideológico.

En este sentido, se han de considerar también las normas editoriales que imperan en el mundo periodístico, que a veces influyen incluso a la hora de redactar la noticia para un destinatario concreto, en este caso, los lectores habituales de The New York Times. Otros criterios que deben tenerse en cuenta son los gramaticales, los pragmáticos, los espaciales, los culturales, los discursivos y todos los derivados de los criterios cognoscitivos que a vista del traductor no es preciso incluir (o, al contrario, es necesario añadir), dado que se presupone que el resultado será un contenido fácil de interpretar para el destinatario meta.

Es evidente que el traductor de esta noticia no solo ha actuado en su rol de traductor, sino también de "trans-editor" (Bielsa, 2010). Esto quiere decir que, en un contexto en el que hay un proyecto de traducción periodística sobre noticias políticas - con los rasgos y sesgos ideológicos que estas puedan tener-, el traductor no es el único que decide sobre la traducción: él se encarga de la parte más lingüística, pero el artículo que se publica finalmente debe llevar el visto bueno de otra persona que actúa como "editora" (en los términos de Bielsa, 2010), que tiene potestad para decidir cómo se publicará la versión definitiva. Esto puede relacionarse con la conclusión a la que llegaron en su momento Ghignoli y Montabes (2014):

Por último, queremos hacer alusión a la fiabilidad de las traducciones en la prensa. Aunque pensemos que todo lo que leemos está bien traducido y es objetivo, es falso. Debemos plantearnos de nuevo la cuestión de por qué no van firmados los textos periodísticos por el traductor. Aunque los traductores, en general, intenten reflejar el contenido del texto origen de la manera más objetiva posible, es prácticamente imposible que el periódico no influya en esta decisión. Además, cabe la posibilidad, y no es remota, de que existan fallos de traducción justificados por el tiempo o no (p. 398).

Los factores ideológicos, políticos y éticos cumplen un gran papel en los ejemplos analizados en este artículo, pues son determinantes para comprender el mensaje del To y para disponer el mismo sentido sobre el TM. Ciertas connotaciones desprendidas del TO no aparecían como tales en el $\mathrm{TM}$, por ejemplo, las marcadas ortotipográficamente con comillas o todas aquellas asunciones de carácter políticamente incorrecto, como se ha analizado. De aquí se desprende que cuando traducimos 
textos de carácter sensible (referido al concepto de sensibilidad de Simms, 1997), a veces marcados por la subjetividad o incluso por la ideología, el traductor tiene la obligación de asegurarse de cuál es la interpretación correcta que hay que dar a determinados enunciados que pueden suponer grandes retos traductológicos, con el objetivo de mantener la fidelidad al texto y de actuar, como mediador lingüístico, de forma objetiva. Esto requerirá de un esfuerzo doble por parte del traductor, puesto que deberá contrastar varias fuentes de información (entre otras, a expertos en el tema o si es posible, al propio autor) durante el proceso de documentación, con el fin de asegurar la ética deontológica del traductor y, en definitiva, que el texto se ha traducido teniendo en cuenta el sentido depositado por el autor en el original y no por la interpretación cognoscitiva del traductor (que podría, en ocasiones, estar influida por otros factores ajenos a la traducción, como se ha indicado anteriormente), razón por la que nos apoyamos, una vez más, en el ACD como método de acercamiento a textos de estas características.

En otras ocasiones, ha sido evidente que el traductor debía contar con conocimientos culturales asociados a la LO, como hemos visto, por ejemplo, en el caso de las paradojas o ironías marcadas en el texto. En estos casos, es importante considerar las normas lingüísticas y estilísticas que pueden delimitar las decisiones traductológicas en el TM, así como todos aquellos aspectos derivados del análisis del ACD, marcados, entre otros, por las relaciones de poder y la manipulación política e ideológica. Dichas relaciones de poder se dirigen especialmente a la sociedad, y el modo de hacer llegar esas pretensiones es por medio del discurso (nivel microestructural), para lo cual será imprescindible acudir a la cognición como interfaz intermedia.

Aunque el resultado final (traducción en LM) no exime de responsabilidad ideológica al procedimiento traductológico llevado a cabo en esta noticia, no han sido ideológicas todas las reformulaciones. Queda claro que la ideología en traducción periodística puede analizarse en cada caso concreto de un modo más exacto si se acude a marcos teóricos y metodológicos como los que hemos utilizado en nuestro modelo de análisis, el cual incorpora el triángulo ideológico del ACD de Van Dijk (1998) para lograr mejores resultados.

\section{Referencias}

Abdi, H. (2019). A skopos-theoretical analysis of political texts from English into Persian: The case of Donald Trump's Executive Orders. Theory and Practice, 9(7), 884-890. http://dx. doi.org/10.17507/tpls.0907.20

Anscombre, J. C. y Ducrot, O. (1983). La argumentación en la lengua. Gredos.

Bassnett, S. y Trivedi, H. (1999). Of colonies, cannibals and vernaculars. En S. Bassnett y $\mathrm{H}$. Trivedi (Eds.), Post-colonial translation: Theory and practice (pp. 95-113). Routledge.

Bastin, G. L., Echeverri, A. y Campo, A. (2004). La traducción en América Latina: propia y apropiada. Estudios: Revista de Investigaciones Literarias y Culturales, 24, 69-94. https://ddd. uab.cat/pub/1611/1611_a2014n8/1611_ a2014n8a7/Georges-Bastin.pdf

Bennoui, A. y Kellou, Y. (2020). Receiving media discourse translation. International Journal of Linguistics, Literature and Translation, 3(4), 1825. http://www.ijllt.org/receiving-media-discourse-translation/

Bielsa, E. (2010). The sociology of translation: Outline of an emerging field. MonTI. Monografias de Traducción e Interpretación, (2), 153-172. https://doi.org/10.6035/MonTI.2010.2.8

Castillo Bernal, M. P. (2016). Spread the news: Translating for the Spanish press in English. En A. M. Rojo López y N. Campos Plaza (Eds.), Interdisciplinarity in translation studies: Some theoretical models, creative approaches and applied methods (pp. 109-122). Peter Lang.

Chomsky, N. (1988). El lenguaje y los problemas del conocimiento. Visor.

Fairclough, N. (2006). Language and globalization. Routledge. 
Davier, L. y Van Doorslaer, L. (2018). Translation without a source text: Methodological issues in news translation. Across Languages and Cultures, 19(2), 241-257. https://doi. org/10.1556/084.2018.19.2.6

Gallardo Camacho, J. (2005). El redactor-traductor en los grandes medios de comunicación con mercados multilingües: caso CNN. Zer, (19), 77-87. https://ojs.ehu.eus/index.php/ Zer/article/view/3842/3424

Ghignoli, A. y Montabes, A. (2014). La traducción y los géneros periodísticos. La traductología en Brasil (2). Mutatis Mutandis. Revista Latinoamericana de Traducción, 7(2), 386-400. https:// revistas.udea.edu.co/index.php/mutatismutandis/article/view/19664/17411

Hernández Guerrero, M. J. (2006). Técnicas específicas de la traducción periodística. Quaderns, (13), 125-139. https://core.ac.uk/download/ pdf/39003465.pdf

Hernández Guerrero, M. J. (2008). La traducción periodística en los diarios españoles de información general. En L. Pegenaute, J. Decesaris, M. Tricás y E. Bernal (Eds.), Actas del III Congreso Internacional de la Asociación Ibérica de Estudios de Traducción e Interpretación. La traducción del futuro: mediación lingüistica y cultural en el siglo XXI (pp. 359-368). http://www.aieti.eu/ wp-content/uploads/AIETI_3_MJHG_Traduccion.pdf

Hernández Guerrero, M. J. (2019). La traducción en las nuevas formas de periodismo. MonTi: Monografias de Traducción e Interpretación, 7293. 10.6035/MonTI.2019.ne5.3

Hurtado Albir, A. (2001). Traducción y traductología. Introducción a la traductología. Cátedra.

Lefevere, A. (2004). Translation, rewriting and the manipulation of literary fame. Shanghai Foreign Language Education Press.

Mason, I. (2009). Discourse, ideology and translation. En M. Baker (Ed.), Critical readings in translation studies (pp. 83-95). Routledge.

Merriam-Webster. (2020). Merriam-Webster dictionary. https://www.merriam-webster.com/

Nida, E. A. (1964). Towards a science of translation with special mention to principles and procedures in Bible translating. Brill.
Padura, L. (2017a, 21 de enero). Trump, un Chevy y la incertidumbre de los cubanos. The New York Times. https://www.nytimes.com/ es/2017/01/21/espanol/opinion/trump-unchevy-y-la-incertidumbre-de-los-cubanos.html

Padura, L. (2017b, 31 de enero). Trump, a Chevy and Cuba's Uncertainty. The New York Times. https:// www.nytimes.com/2017/01/31/opinion/ trump-a-chevy-and-cubas-uncertainty.html

Real Academia Española. (2020). Diccionario de la lengua española (edición del tricentenario). https:// www.rae.es/

Rovena, T. y Marchan, F. (2017). News translation: Text analysis, fieldwork, survey. En S. Hansen-Schirra, O. Czulo y S. Hofmann (Eds.), Empirical modeling of translation and interpreting (pp. 277-310). Language Science Press. https://langsci-press.org/catalog/ view/132/1045/909-1

Saussure, F. de (1916). Curso de lingüística general. Losada.

Schaffner, Ch. (2010). Discourse analysis. En Y. Gambier y L. Van Doorslaer (Eds.), Handbook of translation studies (pp. 235-244). John Benjamins.

Schäffner, Ch. y Bassnett, S. (Eds). (2010). Political discourse, media and translation. Cambridge Scholars Publishing.

Schjoldager, A. (2008). Understanding translation. Academica.

Simms, K. (1997). Translating sensitive texts: Linguistic aspects. Rodopi.

Toury, G. (1985). A rationale for descriptive translation studies. En T. Hermans (Ed.), The manipulation of literature (pp. 16-42). Croom and Helm.

Tymoczko, M. y Gentzler, E. (2002). Translation and power. University of Massachusetts Press.

Van Dijk, T. A. (1996). Opiniones e ideologías en la prensa. Voces y culturas, (10), 9-50. http:// www.discursos.org/oldarticles/Opiniones $\% 20$ e $\% 20$ ideolog\%EDas\%20en $\% 201 a \% 20$ prensa.pdf.

Van Dijk, T. A. (1997). Discurso, cognición y sociedad. Revista Signos. Teoría y práctica de la Educación, (22), 66-74. http://www.discursos. org/oldarticles/Discurso $\% 20$ cognicion $\% 20$ y\%20sociedad.pdf. 
Van Dijk, T. A. (1998). Ideology. A multidisciplinary approach. Sage Publications.

Van Dijk, T. A. (2003). La multidisciplinariedad del análisis crítico del discurso: un alegato en favor de la diversidad. En R. Wodak y M. Meyer (Eds.), Métodos de análisis críticos del discurso (pp. 143-176). Gedisa.

Van Dijk, T. A. (2009). Discurso y poder. Gedisa.

Van Doorslaer, L. (2010). The Belgian conflict frame. The role of media and translation in Belgian political ideologies. En Ch. Schäffner, y S. Bassnett (Eds). (2010). Political discourse, media and translation (pp. 198-211). Cambridge Scholars Publishing.

Wang, D. D. (2019). An analysis of English news translation based on Lefevere's Translation Theory. Beijing University of Posts and Telecommunications.

Zhang, S. y Liu, L. (2019). A study of news translation from the perspective of the manipulation theory. US-China Foreign Language, 17(12), 528-533. https://doi.org/10.17265/1539$8080 / 2019.12 .002$

Cómo citar este artículo: López Ruiz, M. C. (2021). La manipulación en la traducción de noticias del español al inglés. Estudio de caso desde el análisis crítico del discurso. Mutatis Mutandis, Revista Latinoamericana de Traducción, 14(1), 119-141. https://doi.org/10.17533/udea.mut.v14n1a05 